\title{
Immunomodulatory activities of pixatimod: emerging nonclinical and clinical data, and its potential utility in combination with PD- 1 inhibitors
}

Edward Hammond ${ }^{1}$, Nicole M. Haynes ${ }^{2,3}$, Carleen Cullinane ${ }^{2,3}$, Todd V. Brennan ${ }^{4}$, Darryn Bampton ${ }^{1}$, Paul Handley ${ }^{1}$, Tomislav Karoli ${ }^{1,7}$, Fleur Lanksheer ${ }^{5,8}$, Liwen Lin ${ }^{4}$, Yiping Yang ${ }^{6}$ and Keith Dredge ${ }^{1 *}$ (i)

\begin{abstract}
Background: Pixatimod (PG545) is a novel clinical-stage immunomodulatory agent capable of inhibiting the infiltration of tumor-associated macrophages (TAMs) yet also stimulate dendritic cells (DCs), leading to activation of natural killer (NK) cells. Preclinically, pixatimod inhibits heparanase (HPSE) which may be associated with its inhibitory effect on TAMs whereas its immunostimulatory activity on DCs is through the MyD88-dependent TLR9 pathway. Pixatimod recently completed a Phase la monotherapy trial in advanced cancer patients.
\end{abstract}

Methods: To characterize the safety of pixatimod administered by intravenous (IV) infusion, a one month toxicology study was conducted to support a Phase la monotherapy clinical trial. The relative exposure (AUC) of pixatimod across relevant species was determined and the influence of route of administration on the immunomodulatory activity was also evaluated. Finally, the potential utility of pixatimod in combination with PD-1 inhibition was also investigated using the syngeneic 4T1.2 breast cancer model.

Results: The nonclinical safety profile revealed that the main toxicities associated with pixatimod are elevated cholesterol, triglycerides, APTT, decreased platelets and other changes symptomatic of modulating the immune system such as pyrexia, changes in WBC subsets, inflammatory changes in liver, spleen and kidney. Though adverse events such as fever, elevated cholesterol and triglycerides were reported in the Phase la trial, none were considered dose limiting toxicities and the compound was well tolerated up to $100 \mathrm{mg}$ via IV infusion. Exposure (AUC) up to $100 \mathrm{mg}$ was considered proportional with some accumulation upon repeated dosing, a phenomenon also noted in the toxicology study. The immunomodulatory activity of pixatimod was independent of the route of administration and it enhanced the effectiveness of PD-1 inhibition in a poorly immunogenic tumor model.

Conclusions: Pixatimod modulates innate immune cells but also enhances T cell infiltration in combination with anti-PD-1 therapy. The safety and PK profile of the compound supports its ongoing development in a Phase Ib study for advanced cancer/pancreatic adenocarcinoma with the checkpoint inhibitor nivolumab $\left(O p d i v 0^{\oplus}\right)$.

Trial registration: ClinicalTrials.gov Identifier: NCT02042781. First posted: 23 January, 2014 - Retrospectively registered.

Keywords: Pixatimod, PG545, Immunomodulatory, Tumor-associated macrophage, Dendritic cell, NK cell, T cell, PD1 inhibition, Toxicology, Pharmacokinetics, Clinical trial, Pancreatic adenocarcinoma

\footnotetext{
* Correspondence: keith.dredge@zucero.com.au

'Zucero Therapeutics, Brisbane, QLD 4076, Australia

Full list of author information is available at the end of the article
}

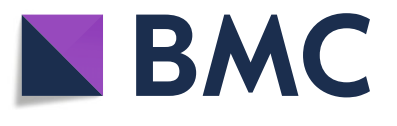

(c) The Author(s). 2018 Open Access This article is distributed under the terms of the Creative Commons Attribution 4.0 International License (http://creativecommons.org/licenses/by/4.0/), which permits unrestricted use, distribution, and reproduction in any medium, provided you give appropriate credit to the original author(s) and the source, provide a link to the Creative Commons license, and indicate if changes were made. The Creative Commons Public Domain Dedication waiver (http://creativecommons.org/publicdomain/zero/1.0/) applies to the data made available in this article, unless otherwise stated. 


\section{Background}

Pixatimod is the international non-proprietary name designated to the compound formerly described as PG545 in the literature [1] and is a cholestanol-sulfotetrasaccharide conjugated small molecule compound (Fig. 1). The oligosaccharide backbone of pixatimod is derived from starch, and retains the amylose structure of $\alpha(1 \rightarrow 4)$-linked glucose residues. Coupling the sulfated oligosaccharide to a lipophilic cholestanol aglycone significantly increased the elimination half-life in vivo, while reducing the unwanted anticoagulant activity associated with similar compounds [2] but retaining the potent inhibition of the heparan sulfate (HS)-degrading enzyme heparanase-1 (HPSE), a key drug target $[1,3,4]$ considered a master regulator of the aggressive cancer phenotype [5-8].

Pixatimod inhibits the infiltration of tumor-associated macrophages (TAMs) $[9,10]$ but, moreover, it also stimulates dendritic cells (DCs) [11]. In terms of its immunomodulatory activity on TAMs, there is preclinical evidence that heparanase may be responsible for this activity [10] and is known to direct the tumor-promoting behaviour of TAMs in pancreatic cancer [12], and promote disease progression in pancreatitis $[13,14]$ and pancreatic cancer [14-16]. The presence of TAMs and M2 macrophages limit immune cell engagement and are associated with decreased survival in pancreatic cancer [17]. However, M1, but not M0 or M2 macrophages, have the ability, not unlike DCs, to prime autologous NK cells and direct $T$ cells $[18,19]$. In addition to the reported activity of pixatimod on TAMs and M2 macrophages $[9,10]$, the compound also exerts a strong immunostimulatory

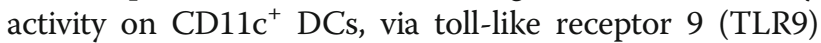
and IL-12 leading to activation of IFN- $\gamma$ producing natural killer (NK) cells [11]. As M1 macrophages also express CD11c, TLR9 and produce IL-12 [20], it is plausible that these myeloid cells play a central role in the activation of innate immunity by pixatimod. Clearly, pixatimod's immunomodulatory effects on these myeloid cells enhance innate immunity and may also drive adaptive immune responses depending on the context (e.g. presence of tumor antigens, combination with PD-1 inhibitors).

Pixatimod has been shown to potently inhibit solid tumor progression and metastasis in a number of syngeneic, orthotopic and xenograft murine models of cancer either alone $[1,10,21-28]$ or in combination

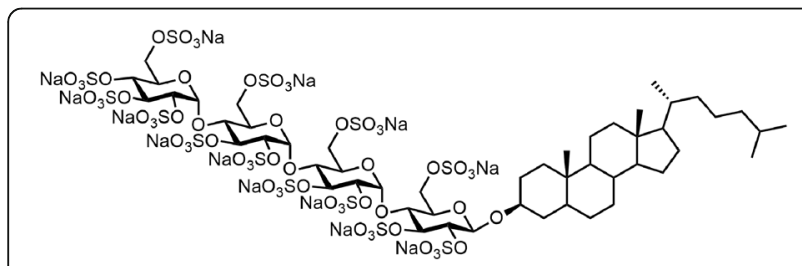

Fig. 1 The structure of pixatimod, formerly known as PG545 with chemotherapy such as paclitaxel or gemcitabine $[29,30]$ and cyclophosphamide with the latter being considered to be mediated by NK cell activation [11]. But its utility in combination with immune checkpoint blockade and the potential to enhance $\mathrm{T}$ cell function or number of infiltrating $\mathrm{T}$ cells into the tumor microenvironment (TME) has not been reported.

Initial clinical development of pixatimod used the subcutaneous route (SC) but switched to intravenous (IV) infusion following local injection site reactions [29]. Subsequently, a non-rodent toxicology study (in beagle dogs) was part of the nonclinical data generated to support the new route of administration which was successfully utilized in a recently completed Phase Ia monotherapy study [31]. An investigational new drug (IND) application was successfully lodged with the USFDA in 2016.

Herein, we describe the new research and development of pixatimod as a once-weekly IV infusion for the treatment of cancer, revealing new data on the proposed mechanism of action, the potential utility of pixatimod in combination with a PD-1 inhibitor, the toxicology and comparative pharmacokinetic profile of pixatimod, and discuss the current clinical and regulatory status of this unique immunomodulatory agent.

\section{Methods}

\section{Nonclinical and clinical safety}

A 1 month toxicology study of pixatimod in beagle dogs was performed under OECD Good Laboratory Practice (GLP) to assess the toxicity and toxicokinetic profile of pixatimod with 5 intravenous, short term (approximately $1 \mathrm{~h}$ ) infusions at three defined dose levels of 2.5, 7.5 and $20 \mathrm{mg} / \mathrm{kg}$ weekly over 29 days. Blood samples were collected for toxicokinetic investigation to provide information on the systemic exposure. Parameters monitored included mortality and morbidity, clinical signs, food consumption, body weight and body temperature, ophthalmoscopy and electrocardiographic measurements. Laboratory investigations were performed using haematology (ADVIA 120), coagulation (AMAX Density Plus Coagulometer), clinical chemistry (VITROS 950) and urinalysis (URYXXON 300). At the end of the treatment, all animals were euthanized and subjected to a complete necropsy with selected organs weighed and followed by a detailed histopathology evaluation. Bone marrow smears from the femur and sternum were prepared at necropsy, fixed and stained with May-Grünwald and Giemsa stain for analysis. Blood samples obtained during the Phase Ia monotherapy study (PG545102) were collected weekly and the parameters reported herein were analysed by local hospital laboratories. 
Nonclinical and clinical bioanalysis and pharmacokinetics Plasma samples were analysed using a LC-MS/MS method as previously described [21]. Pharmacokinetic parameters in animal studies were determined by non-compartmental analyses (NCA) of the mean plasma pixatimod concentration vs time profiles for each dose using the linear trapezoidal method in WinNonlin 5.2.1. For comparison with previous animal studies, exposure data $\left(\mathrm{AUC}_{0 \text {-last }}\right)$ in clinical samples were derived using individual subject NCA type exposure parameters from the original plasma concentration data using $\mathrm{R}$ (64-bit) Version 3.0.1.

\section{Nonclinical efficacy studies}

Female Balb/c mice (6-8 weeks) were obtained from the Walter \& Eliza Hall Institute (Melbourne, Australia). Animal experiments were performed in accordance with institutional guidelines of the Peter MacCallum Cancer Centre. To determine the utility of pixatimod in combination with a PD-1 checkpoint inhibitor antibody (clone RMP1-14 or isotype control antibody 2A3, Bio-X-Cell, $\mathrm{NH}$, USA), mice were inoculated with $1 \times 10^{5} 4 \mathrm{~T} 1.2$ cells into the mammary fatpad. One week later, mice with similar sized tumors (mean tumor volume $56 \mathrm{~mm}^{3}$ ) were randomised into four groups ( $n=6$ mice per group): saline + isotype antibody, pixatimod + isotype antibody, saline + anti-PD-1 antibody and pixatimod + anti-PD-1 antibody. Pixatimod was administered at $15 \mathrm{mg} / \mathrm{kg}$ IP weekly for 3 weeks (days 1, 8 and 15) and anti-PD-1 or isotype antibody $(200 \mu \mathrm{g})$ were given IP on days $1,4,8$, 11 and 15. The experiment was ended on day 18 post treatment initiation (25 days post inoculation) due to emerging toxicities in all treatment groups (e.g. piloerection). Satellite groups of mice ( $n=4$ mice per group) received the same treatments but were euthanized on day 11 for the ex vivo analysis of the immune microenvironment of the 4T1.2 tumors. Spleens were also removed and used as background staining controls.

Immune cells from the collagenase (Collagenase IV, Worthington Biochemical Corporation, NJ, USA) processed tumors and spleens were analysed by flow cytometry using an LSR II analyser (BD Biosciences). Antibodies (ThermoFisher Scientific) used to assess the $\mathrm{T}$ cells and NK cell compartments of the treated 4T1.2 tumors and spleens include CD45.2 (clone 104), TCRb (clone H57-597), CD4 (clone GK1.5), CD8 (clone 536.7), CD44 (clone IM7), CD62L (clone MEL-14), CD69 (clone H1.2F3), CD49b (clone DX5), CD27 (clone LG.7F9), CD335 (clone 29A1.4), DAPI.

To investigate the effect that pixatimod route of administration has upon the activity of this compound, C57BL/6 mice were treated with pixatimod $20 \mathrm{mg} / \mathrm{kg}$ intraperitoneally (IP), intravenously (IV) or subcutaneously (SC) and 2 days later, spleens were isolated to study the activation levels of NK cells for surface expression of CD69 or intracellular expression of IFN- $\gamma$. Antibodies were CD3e (145-2C11), NK1.1 (PK136), CD69 (H1.2F3), IFN- $\gamma$ (XMG1.2), hamster IgG1 isotype (G235-2356) and rat IgG1 isotype (R3-34) were from BD Biosciences (San Jose, CA, USA). Intracellular staining for IFN- $\gamma$ was performed following ex vivo stimulation of splenocytes for $4 \mathrm{~h}$ with $20 \mathrm{ng} / \mathrm{ml}$ PMA and $50 \mathrm{ng} / \mathrm{ml}$ ionomycin in the presence of $5 \mu \mathrm{g} / \mathrm{mL}$ brefeldin A. Intracellular staining for IFN- $\gamma$ was performed following treatment with Cytofix/Cytoperm (BD Biosciences) solution. Flow cytometric data were acquired using a FACSCanto flow cytometer (BD Biosciences), and events were analysed using FlowJo Version 9.9.6 software (TreeStar, Ashland, OR, USA).

\section{Statistical analysis}

In the 4T1.2 model, the percentage tumor growth inhibition was determined according to the following formula: $100 \times(1-\Delta \mathrm{T} / \Delta \mathrm{C})$ where $\Delta \mathrm{C}$ and $\Delta \mathrm{T}$ were calculated by subtracting the mean tumor volume in each group on day 1 of treatment from the mean tumor volume on the day of analysis. Statistical analysis was performed using GraphPad Prism, v 6.0 (GraphPad, La Jolla, CA). An ANOVA analysis was performed followed by Dunnett's post hoc test to compare the tumor growth in the treated groups to the vehicle control. In the dog toxicology study, the analysis was performed using non-parametric Kruskal-Wallis test. The frequency of clinical observations, and necropsy and histopathology findings, was calculated as applicable. For all statistical analyses, statistically significance differences between control and treatment groups was signified by ${ }^{*} P<0.05$, ** $P<0.01, \quad{ }^{* * *} P<0.001,{ }^{* * * *} P<0.0001$ versus vehicle control.

\section{Results \\ Nonclinical and clinical safety}

The toxicity profile of pixatimod in beagle dogs compromised of some findings consistent with that of an immunomodulatory agent. To that end, it is of interest to note that significant elevations in body temperature was apparent following the first dose of pixatimod but the effect appeared to dissipate upon repeat dosing by day 30 (Fig. 2a). The other striking effect was the significant increases in large unstained cells (LUCs) following exposure to pixatimod (Fig. 2b). Despite the changes in LUCs, absolute WBC counts and main subsets (lymphocytes, neutrophils and monocytes) remained within normal ranges though changes did, at times, reach statistical significance (Additional file 1). However, significant changes in APTT, cholesterol, triglycerides and AST were noted in the toxicology study (Fig. 3a-d). These toxicology findings were somewhat consistent with 


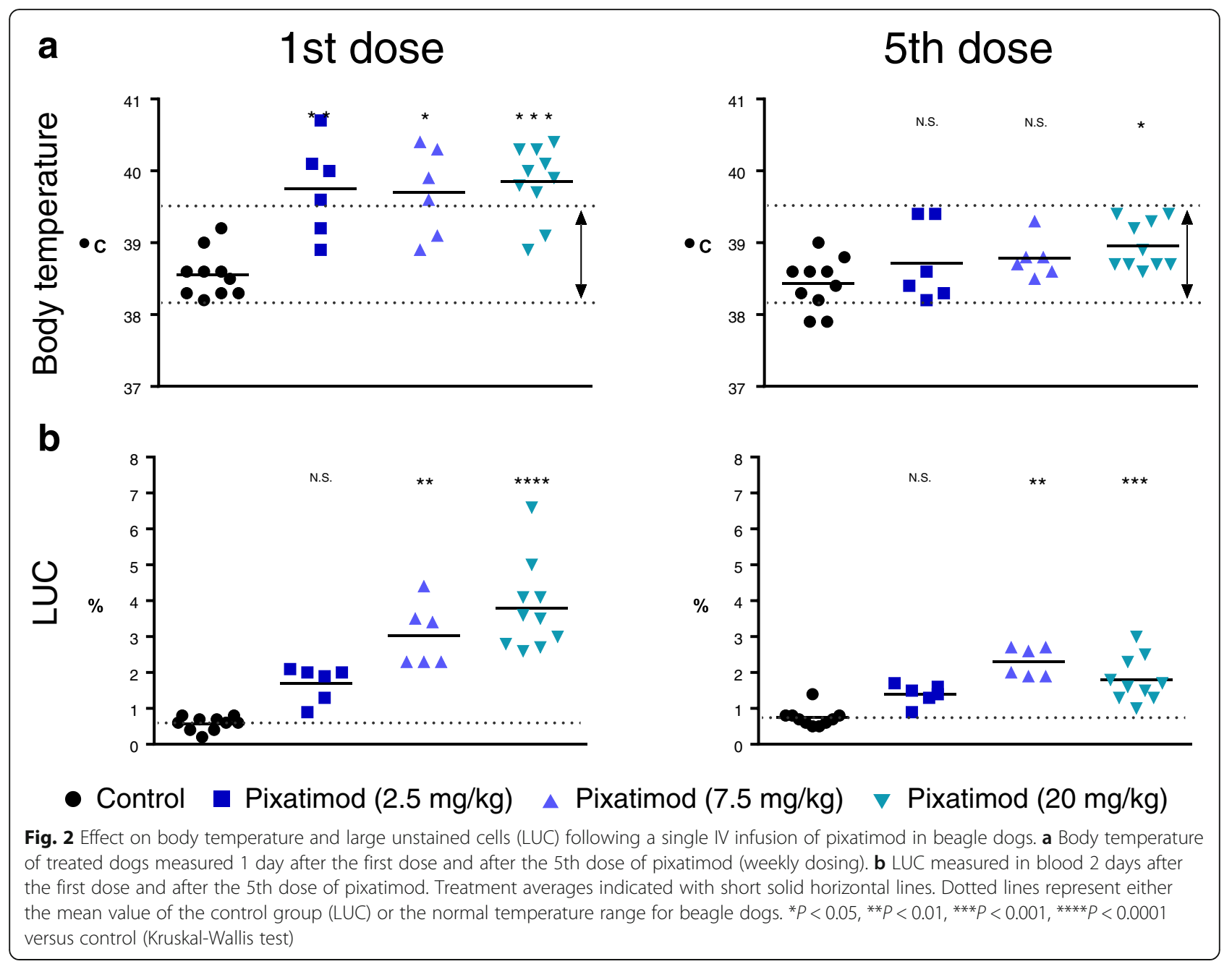

reported adverse events in advanced cancer patients (Fig. 3a-d), though elevations in AST were only prominent in two subjects at the maximum tolerated dose (MTD) in some patients but these were unrelated to pixatimod treatment. In the Phase Ia monotherapy clinical trial, some parameters, such as cholesterol and triglycerides, normalised despite repeated exposure to pixatimod suggesting an adaptive response to treatment. Adverse events, severe adverse events and dose limiting toxicities associated with pixatimod treatment in humans have been previously reported [31].

In the toxicology study, there was no mortality or significant changes in bodyweight gain, food consumption, urinalysis, ophthalmologic investigations, ECGs, heart rate, or any local sign at infusion sites during the study (except for oedema in one high dose individual) associated with pixatimod treatment. Pixatimod significantly increased relative weights of liver and kidneys, with modest but dose-dependent decreases in thymus weights but no effect on spleen weight (Additional file 2A-D). Given spleen weights increase following SC dosing in rodents
(Additional file 2E), there may be a species difference although the influence of the route of administration cannot be fully discounted as a previous toxicology study in beagles reported non-statistically significant increases in relative spleen weights following treatment with pixatimod (Additional file 2F). Treatment also led to microscopic findings at infusion sites, kidneys, liver, thymus and spleen (Additional file 3). Generally speaking, these dose-dependent alterations were characterized as chronic-active inflammation (the coexistence of chronic inflammation with the presence of mononuclear cell population and superimposed by an acute inflammation with polymorphonuclear cells). Pixatimod also induced dose-dependent hypertrophy of Kupffer cells in the liver and inflammatory changes in the kidneys (multifocal perivascular, mixed cellular infiltrate) with glomerular vacuolation and/or sclerosis noted in high dose groups only. Diffuse mixed cellular infiltrate in the spleen and minimal to mild lymphoid atrophy of the thymus were also observed in mid and high dose animals. No treatment-related changes were reported in the bone marrow smears. 
Nonclinical (5th dose)

a

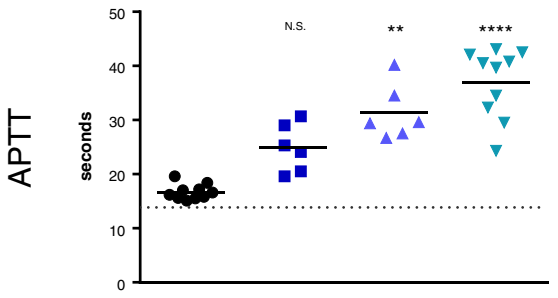

b

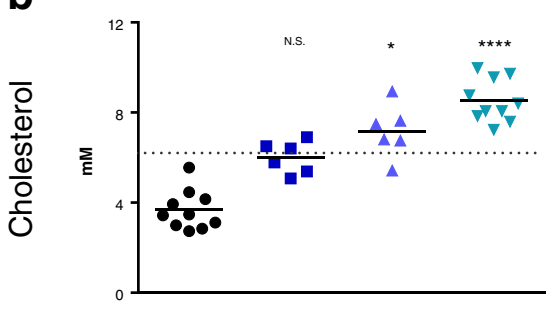

C
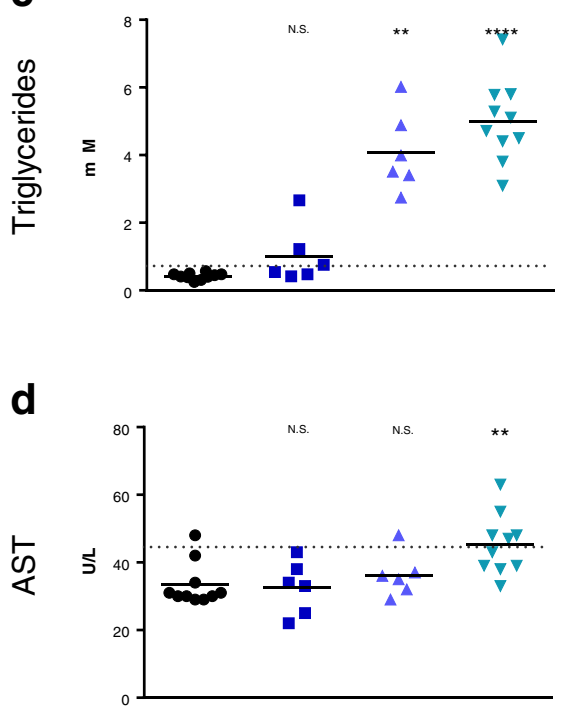

- Control

- Pixatimod $(2.5 \mathrm{mg} / \mathrm{kg})$

Pixatimod $(7.5 \mathrm{mg} / \mathrm{kg})$

$\checkmark$ Pixatimod $(20 \mathrm{mg} / \mathrm{kg})$
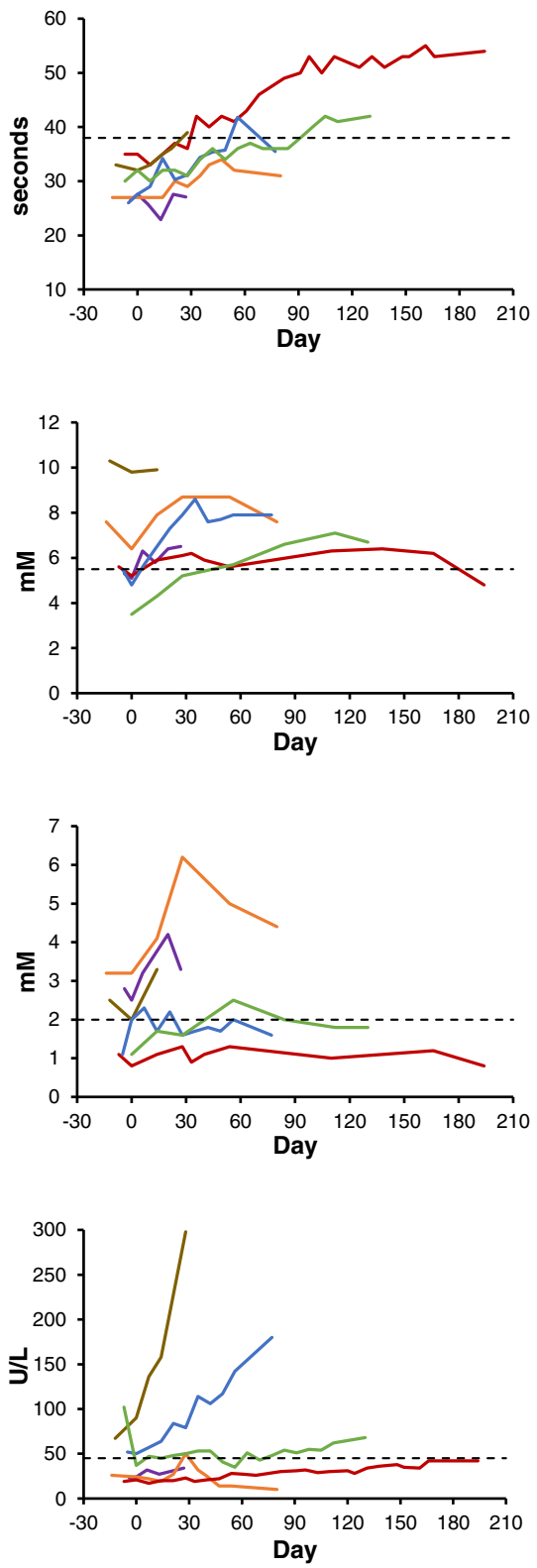

$$
\begin{array}{rrr}
1041 & -3042 & -3043 \\
-3044 & -3045 & -3046
\end{array}
$$

Fig. 3 Effect on APTT, blood lipids and AST of weekly IV dosing in beagle dogs and humans (patients in the $100 \mathrm{mg}$ cohort). APTT (a), cholesterol (b), triglycerides (c) and AST (d) were measured in the blood of dogs after 5 pixatimod doses (weekly dosing). Treatment averages indicated with short solid horizontal lines. Corresponding data from the six $100 \mathrm{mg}$ patients for these 4 parameters are presented over time. Dotted lines represent upper limit of the normal range for each parameter. ${ }^{*}=P<0.05,{ }^{* *} P<0.01,{ }^{* * *} P<0.001,{ }^{* * *} P<0.0001$ versus control (Kruskal-Wallis test)

Comparative exposure (AUC) and route of administration Population-based PK analysis and NCA parameter estimates was reported in the Phase Ia monotherapy study (PG545102) with proportional exposure up to $100 \mathrm{mg}$ dose [31]. Herein, exposure (AUC) following pixatimod in the mouse, dog and in advanced cancer patients is reported in Table 1. Plotting the exposure data from Week 1 from all species (mouse, dog and human) as a function of human equivalent dose (HED) reveals a linear response with a linear regression passing close to the origin (Fig. 4). 
The $\mathrm{AUC}_{0 \text {-last }}$ exposure data for week 4 in patients confirm the accumulation previously reported [31] and is consistent with findings in the dog toxicology study. However, it is unclear whether this is the case in mice as it was impossible to obtain plasma in week 4 (due to the tumor burden in the A2780 xenograft model) and previous data on exposure upon repeated dosing in tumor-bearing immunocompetent mice were not evident across all dose levels, the exposure (AUC) required to achieve efficacy as a monotherapy was $687 \mu \mathrm{g} . \mathrm{h} / \mathrm{mL}$ [21] which is in the range of the low dose group $(2.5 \mathrm{mg} / \mathrm{kg})$ in the toxicology study and the low dose group $(25 \mathrm{mg})$ in the clinical study by the end of the first cycle (1 month of weekly IV treatment).

In addition to assessing the relationship between dosing and exposure across these species, the efficacy of pixatimod was also examined as a function of the route of administration. After dosing mice via IP, IV and SC routes, pixatimod increased the expression of IFN- $\gamma$ and CD69 on NK cells to a similar magnitude irrespective of the route, indicating that pixatimod's immunomodulatory activity is not limited to a particular route of administration (Fig. 5).

\section{Combination therapy potential}

Given the multiple modes of immune evasion that cancers have evolved, a significant clinical effort has commenced to evaluate immunotherapies in combination to increase response rates and broaden the types of cancers that can be treated [32]. Even the most successful immunotherapeutic strategy, targeting PD-1, has shown limited utility as a single agent and yields better patient outcomes by utilizing combination strategies [33]. The syngeneic breast cancer model 4T1.2 is considered poorly immunogenic, highly metastatic, and exhibits limited responsiveness to checkpoint blockade [34]. To assess the clinical potential of the pixatimod

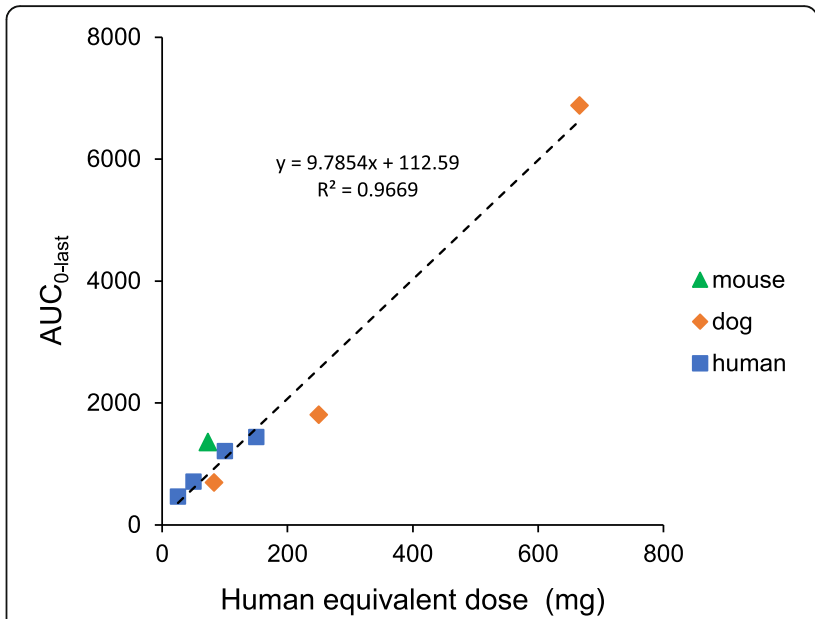

Fig. 4 The relationship between a single pixatimod dose and exposure (AUC) across mouse, dog and human. The Week 1 AUC $_{0-}$ last data from Table 1 are plotted as a function of the human equivalent dose (HED). The dotted line represents a linear regression of all of the data yielding an $R^{2}$ of 0.9669 and a y intercept of 112.59

and anti-PD-1 antibody combination regimen, these agents were tested in the 4T1.2 breast model (Fig. 6a). The combination was significantly more efficacious than the control group or anti-PD-1 treatment alone. Tumor growth in the pixatimod plus isotype antibody (Pixatimod), vehicle plus anti-PD-1 antibody (Anti-PD1) and pixatimod plus anti-PD-1 (Combination) groups was inhibited by 68,44 and $84 \%$, respectively on day 18 . Tumor growth in the pixatimod and combination groups was significantly inhibited on day 18 compared to the vehicle plus isotype antibody (Control) group (Fig. 6b).

The tumors of satellite groups from this study were analysed at day 11 of the study for immune cell populations and activation status. The effect of the combination treatment upon intratumoral immunity was striking. The frequency of both $\mathrm{CD} 4^{+}$(Fig. 7a) and $\mathrm{CD} 8^{+}$

Table 1 Comparison of mean exposure (AUCO-last) in mouse, dog and human based following intravenous administration of pixatimod

\begin{tabular}{|c|c|c|c|c|}
\hline Species & Dose $(\mathrm{mg} / \mathrm{kg})$ & HED $^{\text {a } / \text { human dose }(\mathrm{mg})}$ & $\mathrm{AUC}_{\text {0-last }}(\mu \mathrm{g} \cdot \mathrm{h} / \mathrm{mL})^{\mathrm{b}}$ Week 1 & $\mathrm{AUC}_{0 \text {-last }}(\mu \mathrm{g} \cdot \mathrm{h} / \mathrm{mL})^{\mathrm{b}}$ Week 4 \\
\hline Mouse & 15 & 73 & $1358^{c}$ & N.D. \\
\hline \multirow[t]{3}{*}{ Dog } & 2.5 & 83 & 697 & 1372 \\
\hline & 7.5 & 250 & 1809 & 4936 \\
\hline & 20 & 666 & 6883 & 10,536 \\
\hline \multirow[t]{4}{*}{ Human } & - & 25 & 465 & 610 \\
\hline & - & 50 & 709 & 1688 \\
\hline & - & 100 & 1209 & 2381 \\
\hline & - & 150 & 1441 & 2781 \\
\hline
\end{tabular}

${ }^{a}$ HED - Human equivalent dose conversion (https://www.fda.gov/downloads/drugs/guidances/ucm078932.pdf)

${ }^{\mathrm{b}}$ Area under the pixatimod concentration-time curve from time $0 \mathrm{~h}$ (relative to applicable dose) to the last measureable concentration over the dosing interval; derived from the measured concentration values using linear trapezoidal summation

cOriginally published in [29]

N.D. Not Determined 


\section{a}
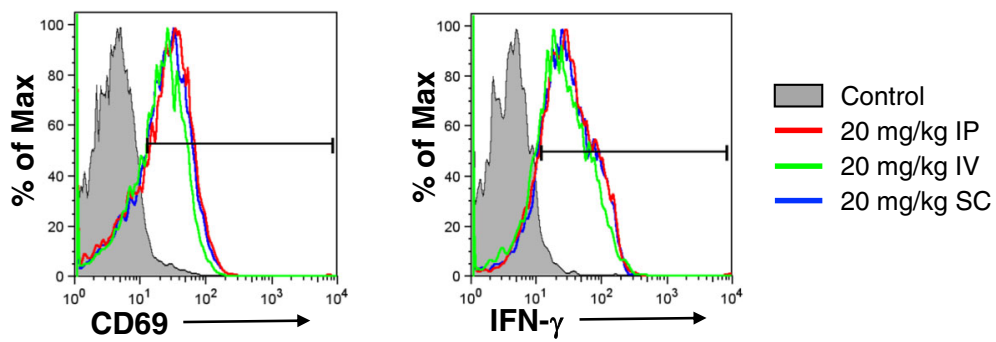

b
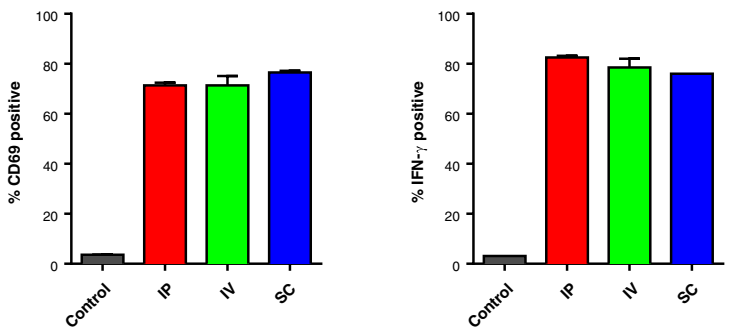

Fig. 5 The effect that route of pixatimod administration has on the activation of NK cells. a Mice were dosed with $400 \mu \mathrm{g}$ (20 mg/kg) of pixatimod intraperitoneally (IP), intravenously (IV) or subcutaneously (SC) and after 2 days the activation status of NK cells (CD3- ${ }^{-}$NK1.1 ${ }^{+}$) isolated from spleens were assessed. b The percentage of NK cells positive for CD69 and IFN- $\gamma$ are plotted. The gating for CD69 and IFN- $\gamma$ positive NK cells is shown in panel a

(Fig. 7d) T cells were significantly elevated in the tumors of the combination group. Within both the CD4 and CD8 populations, effector memory and central memory cells were increased by the pixatimod-anti-PD-1 combination (Fig. 7b-f). Moreover, both the frequency of bulk NK cells and activated $\mathrm{CD}^{+} 9^{+} \mathrm{NK}$ cells were also increased in tumors of the combination group (Fig. $7 \mathrm{~g}$ and $\mathrm{h}$ ). In contrast, there was little or no increase in the abundance of $\mathrm{CD}^{+}$or $\mathrm{CD}^{+} \mathrm{T}$ cells or NK cells in the spleens of the mice treated with pixatimod, anti PD-1 antibody or the combination when compared to the spleens of control mice indicating that the immune response was tumor-specific (Additional file 4).

\section{Discussion}

Pixatimod has a unique mechanism of action and a structure that is similarly unconventional in the pharmaceutical world. It targets TAMs via the inhibition of heparanase $[9,10]$ and it also activates NK cells through TLR9-dependent stimulation of DC [11]. The unique activity is not only attributed to its heparan sulfate (HS) mimetic structure - a recognized approach in the generation of heparanase inhibitors [5-8] - but importantly, to its lipophilic moiety (cholestanol), which sets pixatimod apart from other HS mimetics [1, 3, 21]. The resultant compound is more polar and larger than a typical small molecule drug but has been demonstrated to possess potent antitumor activity in multiple cancer models indicating potential utility in a range of cancers, particularly in combination with other therapeutics. An example of this utility is shown by the efficacy of pixatimod when combined with an anti PD-1 antibody in the 4T1.2 model (Fig. 6), the \%TGI for the combination group (84\%) was almost two-fold higher compared with the anti-PD-1 antibody group (44\%). Though the impact of the combination on 4T1.2 metastasis was not assessed, pixatimod has been previously demonstrated to inhibit spontaneous metastasis and enhance overall survival in this model [22].

In terms of an immune response, synergy was observed in the pixatimod and anti-PD-1 treatment group as significant increases in both tumor-specific CD8 and CD4 effector memory and central memory T cells were evident. Moreover, the combination significantly increased NK cell numbers in the tumors. Though pixatimod alone didn't significantly increase intratumoral NK cells as previously reported [11], this could be attributed to the different NK-characterizing antibodies (CD49b and CD27 in the 4T1.2 data versus CD335 in the A20 data in [11]) which also may detect different NK cell subsets [35]. Moreover, there may be differences in pixatimod's ability (as a monotherapy) to increase NK cell infiltration into primary tumors which are disseminated or 'diffuse' such as lymphomas compared with transplantable carcinoma models. Finally, pixatimod possesses potent anti-metastatic activity in the $4 \mathrm{~T} 1.2$ model [22], so it is conceivable that activated NK cells 


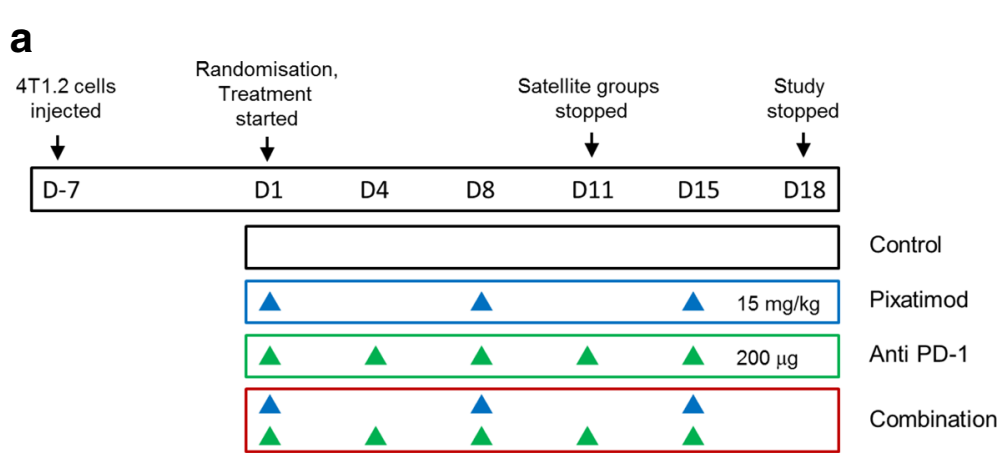

b

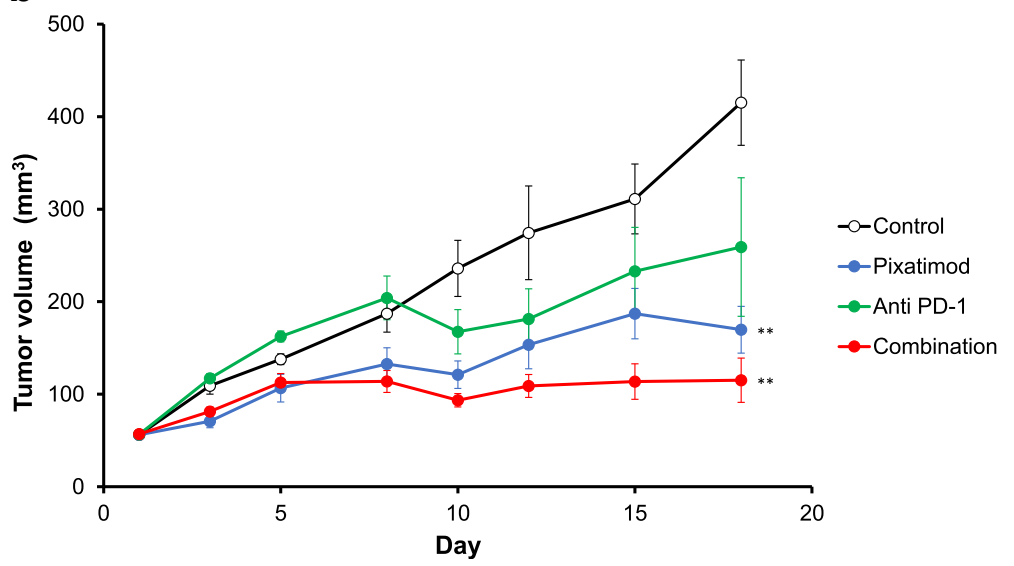

Fig. 6 Efficacy of pixatimod in combination with anti-PD-1 in a syngeneic 4T1.2 breast cancer mouse model. a Seven days after inoculation, mice were randomised into four treatment groups of six mice each: Control (PBS weekly plus $200 \mu \mathrm{g}$ isotype antibody twice weekly); Pixatimod (pixatimod $15 \mathrm{mg} / \mathrm{kg}$ weekly plus isotype antibody twice weekly); Anti-PD-1 (PBS weekly plus $200 \mu \mathrm{g}$ anti-PD-1 antibody twice weekly) and Combination (pixatimod weekly plus anti PD-1 antibody twice weekly). Satellite groups of four mice were treated identically and were stopped at day 11 for immune cell analysis (Fig. 6). b Tumor volumes were measured throughout the study and the means compared at study conclusion (day 18). ${ }^{* *} P<0.01$ versus control

could be directed to distant metastatic sites rather than accumulate in the primary tumor, especially given the critical role of NK cells in the control of spontaneous metastasis in the 4T1.2 model [36].

The key message from this study is that pixatimod, in combination with a PD1 inhibitor, has the potential to enhance a tumor-specific $\mathrm{T}$ cell response capable of inhibiting tumor growth, a notion which holds great potential for cancer treatment [37-39]. A number of innate immune activators (including TLR9 agonists) are under development and could prove to be complimentary to $\mathrm{T}$ cell based therapies but typically they are administered locally which could limit their utility in the clinic [40, 41]. Thus, pixatimod offers an alternate approach to promote T cell- (and NK cell-) based inflammation in non-inflamed tumors, which is considered one of the biggest challenges in order to expand the subset of patients in whom currently active immunotherapies appear effective [42, 43].

The mechanism whereby the pixatimod/anti-PD-1 combination promotes the recruitment of $\mathrm{T}$ cells into tumors is not clear. Pixatimod alone has been shown to activate NK cells which can be detected in spleens and tumors in mouse models but in the same study it showed no activation or recruitment of $\mathrm{T}$ cells [11]. However, in combination with anti-PD-1 antibody, pixatimod significantly increases the infiltration of tumor-specific $\mathrm{T}$ cells into the TME. This could be related to the blockade of TAMs via pixatimod-mediated heparanase inhibition $[9,10]$ or maturation of plasmacytoid DCs (pDCs) via TLR9 [44] resulting in the diminution of the tolerogenic signalling environment associated with TAM, other myeloid cells or immature pDCs, particularly in cold tumors. Though the first mechanism could lead to synergy with anti-PD1 agents due to reduced numbers of immunosuppressive PD-L1/ $2^{+}$cells in the TME, such as tolerogenic myeloid cells or tumor cells, the second mechanism via TLR9 [11] is equally, if not more likely to work in concert with PD-1 blockade. TLR9 agonists are known to increase the efficacy of anti-PD-1 agents in preclinical models $[45,46]$ and can involve the polarization of naive macrophages 
a

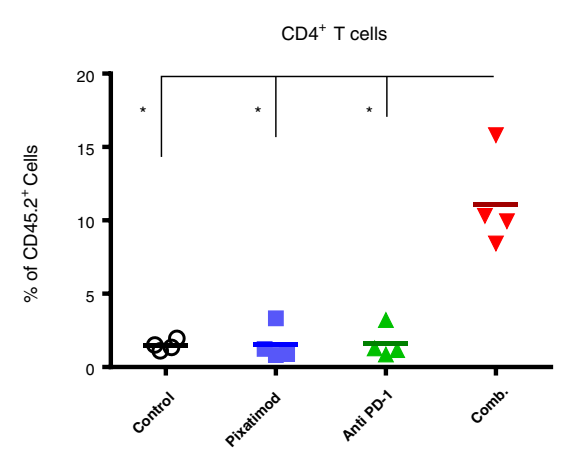

b

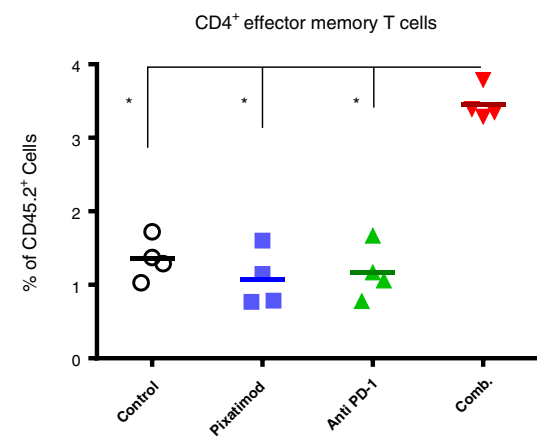

C

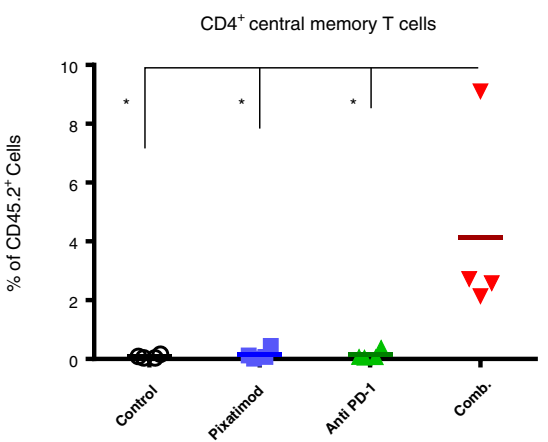

d

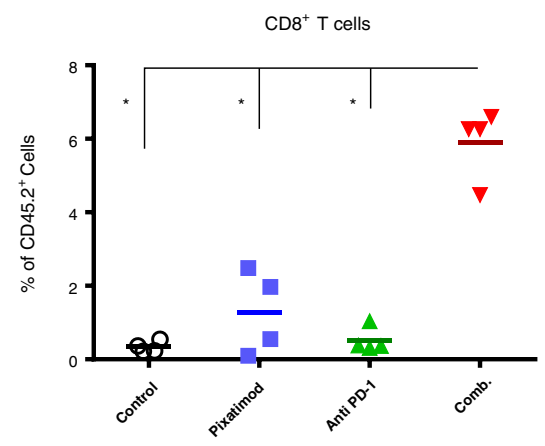

e

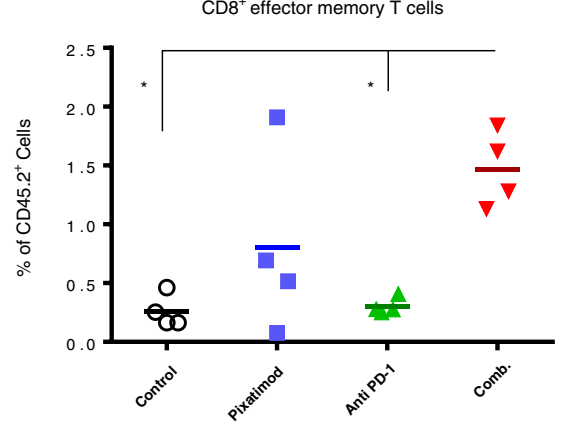

f

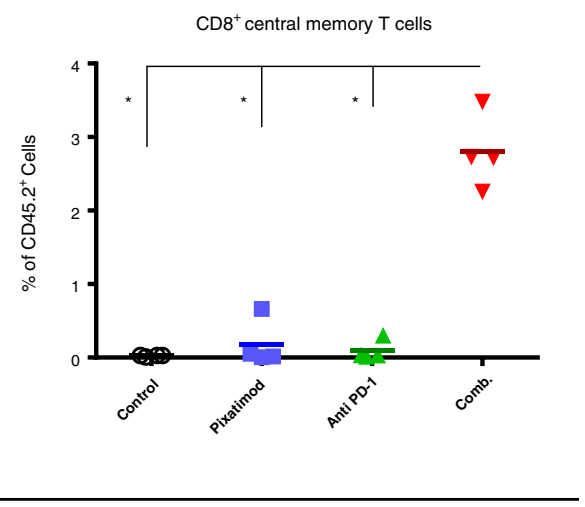

h

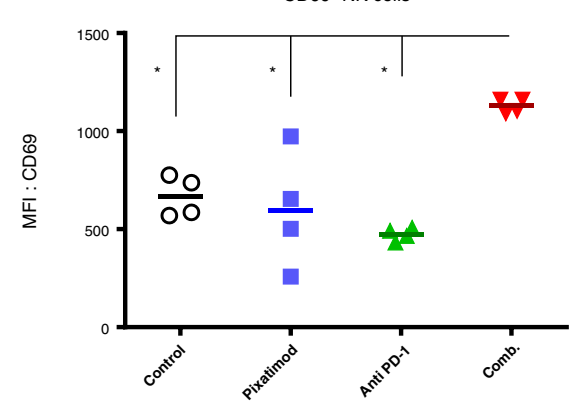

Fig. 7 (See legend on next page.) 
(See figure on previous page.)

Fig. 7 Phenotype analysis of immune cells in the tumors of the 4T1.2 breast cancer model (Fig. 6). Immune cells were isolated from the tumors of mice in the satellite groups (day 11) and phenotyped by flow cytometry. a Total CD4 ${ }^{+} T$ cells (b) effector memory $C D 4^{+} T$ cells and (c) central memory CD4 ${ }^{+} T$ cells. $\mathbf{d}$ Total CD8 ${ }^{+} \mathrm{T}$ cells $(\mathbf{e})$ effector memory CD8 ${ }^{+} \mathrm{T}$ cells and (f) central memory $\mathrm{CD} 8^{+} \mathrm{T}$ cells. $\mathbf{g}$ Total and (h) CD69 ${ }^{+} \mathrm{NK}$ cells. Treatment averages indicated with short solid horizontal lines. ${ }^{*} \mathrm{P}<0.05$ versus control

toward a M1-like phenotype [47]. Conversely, it is known that depletion of TAMs or M2 macrophages using CSF-1R inhibition enhances DC immunotherapy [48] and checkpoint inhibition [49]. Viewing M1 macrophages and DC as essentially antigen presenting cells [19], we conclude that at least in combination with a PD-1 inhibitor, pixatimod's immunomodulatory activity (whether this is a direct stimulation of DC or a polarization from M2 to M1 macrophages) leads to improved immune recognition of tumor cells as shown by the synergistic increases in T cell infiltration into the TME.

Given the potent immune stimulatory activity of pixatimod, it is important to characterize toxicologic responses that could be associated with excessive activation of the immune system. Upon initial exposure to pixatimod in beagle dogs, the elevations in body temperature and LUCs are particularly noteworthy. Flu-like symptoms (including fever) have been previously reported as a response to innate immune activators such as TLR9 agonists [44] but in this instance at least, elevated temperatures dissipated upon repeated dosing suggesting an adaptive response. Similarly, pixatimod-induced elevations in percent LUCs - defined as atypical large lymphocytes or monocytic cells that may increase with an inflammatory response [50] - declined, at least in the high dose group, upon repeated exposure. In the PG545102 monotherapy trial, pixatimod induces flu-like symptoms in patients at doses at or above $50 \mathrm{mg}$, which required prophylactic paracetamol [31], but LUCs could not be measured at hospital sites.

The major nonclinical toxicities associated with pixatimod were elevated cholesterol and triglyceride levels, increases in relative weights of liver and kidney, cellular infiltrates in liver, kidney, and spleen, hypertrophy of Kupffer cells, tubular dilatation and glomerular vacuolation and/or sclerosis. Though relative spleen weights significantly increase following pixatimod treatment in rodents following SC dosing, this finding was not significant in the SC dog toxicology study and not apparent whatsoever in the IV dog study. So, while TLR9 expression may be lower in dog or human macrophages than mouse or rat macrophages [51], the route of administration could also contribute to this effect. This is potentially relevant for two reasons. First, given that pixatimod's immunostimulatory may be mediated via TLR9 [11], but note that pixatimod is not a CpG oligonucleotide (ODN) or a TLR9 agonist, this may also account for the fact that the histopathological findings for CpG-ODN [51] were not reported in the pixatimod toxicology studies. Second, the clinical route of administration is now via the IV route and though the safety profile appears promising, the immunostimulatory effect on NK cells is equivalent to other route of administration. In addition to the hyperlipidemia and vacuolation, other changes such as decreased RBC, HGB, HCT, lymphocytes and platelets and increases in percent neutrophils and APTT were considered toxicologically relevant. In contrast, these parameters were not clinically significant in patients though hyperlipidema and elevated APTT were considered to be related to pixatimod treatment. Taken together, the safety profile of pixatimod is consistent with an innate immune activator which has the potential to induce an inflammatory response in the host.

The PK profile of pixatimod has been previously described in mouse [29] and human subjects [31] but herein the relationship between pixatimod dose and exposure (after the first dose) was found to be linear across mouse, dog and human (Fig. 4). This indicates, firstly, that exposure, at least for the initial pixatimod dose, is predictably proportional to the dose administered and, secondly, that the assumptions inherent in extrapolation from animal efficacy and toxicology studies to humans appear valid and may be utilised for continuing PK/PD analyses during development.

In the Phase Ia monotherapy trial, 20 patients experienced treatment emergent adverse events (AE) that were possibly, likely, or certainly related to pixatimod. The majority of AEs in this category were associated with infusion reactions: chills, pyrexia, infusion related reactions, and hypertension. In terms of clinical activity, 16 patients had efficacy assessments during pixatimod treatment and six of these had stable disease (SD) at 8 weeks as measured by RECIST 1.1 criteria. This ratio, six of sixteen assessed, represents a $38 \%$ disease control rate at 8 weeks. The estimated median duration of SD for patients on the study was 57 days [31]. Based on the immunomodulatory properties of pixatimod and emerging preclinical data in combination with an anti-PD-1 antibody, a new clinical trial is underway in Australia investigating pixatimod in combination with nivolumab $\left(\mathrm{Opdivo}^{\circ}\right)$ in patients with advanced solid tumors with an expansion cohort in patients with pancreatic adenocarcinoma.

\section{Conclusions}

Pixatimod modulates macrophages and DCs to activate NK cells but in this study it has also been demonstrated 
to enhance the antitumor activity of a PD-1 inhibitor, an effect that correlates with increased frequency of $\mathrm{T}$ cells and NK cells within the TME. The safety profile indicated that it has mild inflammatory properties but the compound was well tolerated up to $100 \mathrm{mg}$ in the monotherapy clinical trial. Pixatimod is currently under investigation in a Phase Ib study for advanced cancer/ pancreatic adenocarcinoma with the checkpoint inhibitor nivolumab $\left(\mathrm{Opdivo}^{\circ}\right)$.

\section{Additional files}

Additional file 1: Effect on total white blood cells (WBC), lymphocytes, neutrophils and platelets of weekly IV dosing in beagle dogs and humans (patients in the $100 \mathrm{mg}$ cohort). WBC (A), lymphocytes (B), neutrophils (C), monocytes (D) and platelets (E) were measured in the blood of dogs after 5 pixatimod doses (weekly dosing). Treatment averages indicated with short solid horizontal lines. Corresponding data from the six $100 \mathrm{mg}$ patients for these 4 parameters are presented over time. Dotted lines represent limits of the normal range for each parameter. ${ }^{*} P<0.05,{ }^{* *} P<0.01,{ }^{* *} P<0.001,{ }^{*}{ }^{* *} P<0.0001$ versus control (Kruskal-Wallis test). (PPTX $142 \mathrm{~kb}$ )

Additional file 2: Effect of pixatimod dosing on selected organ weights in beagle dogs (IV and SC) and rats (SC). Repeated weekly IV exposure ( $X$ 5) of pixatimod increases the relative weights of liver (A) and kidney (B) but not thymus (C) nor spleen (D) in beagle dogs. Relative weights of liver and kidneys were significantly increased in the high dose group (20 mg/kg) with the mid-dose group ( $7.5 \mathrm{mg} / \mathrm{kg}$ ) also significantly increasing the relative weight of kidneys. Data shown is for combined sexes. Relative spleen weight was increased in a dose-dependent manner after repeated weekly SC exposure $(X 5)$ in rats (E). Study size, $n=15$ for control and $48 \mathrm{mg} / \mathrm{kg}$ groups and $n=10$ for 3 and $12 \mathrm{mg} / \mathrm{kg}$ groups. As seen with IV exposure (D), repeated weekly SC exposure $(\times 5)$ in beagle dogs had no significant effect on spleen weight (F). Study size, $n=5$ for control and $20 \mathrm{mg} / \mathrm{kg}$ groups and $n=3$ for 2.5 and $7.5 \mathrm{mg} / \mathrm{kg}$ groups. ${ }^{*} P$ $<0.05$, ${ }^{* *} P<0.01,{ }^{* * *} P<0.001$ versus control (Kruskal-Wallis test for the dog study, ANOVA followed by Dunnett's comparison for the rat study). (PPTX $65 \mathrm{~kb}$ )

Additional file 3: The incidence and severity of noteworthy microscopic findings in kidneys, liver, spleen and thymus. Perivascular mixed cell infiltrate was present in most mid- and high-dose individuals. Minimal to mild dilatation of renal tubules was apparent in most treated individuals though minimal or mild glomerular vacuolation or sclerosis was only reported in high-dose individuals. Minimal to mild hepatocellular hypertrophy was evident across dose levels whereas incidence and severity of hypertrophy of Kupffer cells in the liver was dose-dependent. A minimal to mild increase in cell infiltrate was apparent some high-dose individuals. There was also evidence of diffuse mixed cellular infiltrate in the spleen and minimal to mild lymphoid atrophy of the thymus observed in mid and high dose animals. (DOCX $13 \mathrm{~kb}$ )

Additional file 4: Phenotypic analysis of $\mathrm{CD}^{+}$and $\mathrm{CD}^{+} \mathrm{T}$ cells, and $\mathrm{NK}$ cells, in the spleens of the 4T1.2 breast cancer model. Splenocytes were isolated in the satellite groups (day 11) and assessed by flow cytometry. (A) Total CD4 ${ }^{+} \mathrm{T}$ cells (B) Total CD8 ${ }^{+} \mathrm{T}$ cells (C) Total NK cells. Spleens of all four mice from each treatment group were combined for analysis. (PPTX $47 \mathrm{~kb}$ )

\section{Abbreviations}

AE: Adverse event; APTT: Activated partial thromboplastin time; AST: Aspartate transaminase; AUC: Area under the curve; DC: Dendritic cells; FDA: Food and Drug Administration; HCT: Hematocrit; HED: Human equivalent dose; HGB: Hemoglobin; IND: Investigational new drug; IP: Intraperitoneal; IV: Intravenous; LUC: Large unstained cells; MTD: Maximum tolerated dose; NK (cells): Natural killer cells; PK: Pharmacokinetics; RBC: Red blood cells; RECIST: Response evaluation criteria in solid tumors;
SC: Subcutaneous; SD: Stable disease; TAM: Tumor-associated macrophages; TME: Tumor micro-environment; WBC: White blood cells

\section{Acknowledgements}

Our appreciation to the oncologists involved in the Phase I monotherapy trial (Prof Michael Millward, Sir Charles Gardiner Hospital, Perth; Prof Michael Brown, Royal Adelaide Hospital, Adelaide; Dr. Jason Lickliter, Nucleus Network, Melbourne), Linda Wright and Michael Vaughan at TetraQ for the bioanalysis work, Monika Petus and David Esdaile at CiToxLAB for the toxicology study, Glynn Morrish at Clinical Network Services, Brisbane for the pharmacokinetic analyses. We thank Kerry Ardley at the Peter MacCallum Cancer Centre for expert technical assistance.

\section{Availability of data and materials}

Due to confidentiality agreements with research collaborators and service providers, supporting data can only be made available to bona fide researchers subject to a non-disclosure agreement.

\section{Authors' contributions}

$\mathrm{EH}, \mathrm{DB}$ and $\mathrm{KD}$ were the main investigators and take primary responsibility for the paper. TB, LL, YY performed the in vivo experiment exploring pixatimod route of administration. EH, FL, KD, CC and $\mathrm{NH}$ designed and/or conducted the 4T1.2 model and tumor infiltration analysis. PH and TK manufactured pixatimod, and along with DB and FL participated in the design and coordination, and helped to draft the manuscript. KD was Sponsor's Monitor for GLP toxicology and toxicokinetic studies. EH, KD and DB designed and analysed aspects of the pharmacokinetic and clinical pathology data presented herein, including from the PG545102 clinical trial. $\mathrm{EH}$ and $\mathrm{KD}$ wrote the paper. All authors read and approved the final manuscript.

\section{Ethics approval}

For the route of administration studies in mice, experiments were done in accordance with protocols approved by the Animal Care and Use Committee of Duke University. The 4 T1.2 syngeneic tumor model study was performed with approval of the Peter MacCallum Cancer Centre Animal Experimentation Ethics Committee.

For the toxicology study, procedures involving the care and use of animals in this study were reviewed and approved by the Institutional Animal Care and Use Committee (IACUC). During the study, the care and use of animals were conducted in accordance with the relevant principles currently used with regard to animal welfare. CiToxLAB Hungary Ltd.'s facility is AAALAC accredited. The PG545102 study was approved by local human research ethics committees (HREC), the Royal Adelaide Hospital Research Ethics Committee (for RAH site) and Bellberry Limited (for the sites at Linear and Nucleus Network). The trial was conducted in accordance with the ICH Good Clinical Practice (GCP) guidelines and the Declaration of Helsinki. All patients provided written informed consent.

\section{Competing interests}

$\mathrm{EH}, \mathrm{PH}, \mathrm{DB}, \mathrm{TK}, \mathrm{FL}$ and $\mathrm{KD}$ were or are employees of Progen Pharmaceuticals or Zucero Therapeutics. DB receives consultancy fees from Zucero Therapeutics. NMH, CC, TVB, LL and YY declare that they have no competing interests.

\section{Publisher's Note}

Springer Nature remains neutral with regard to jurisdictional claims in published maps and institutional affiliations.

\section{Author details}

'Zucero Therapeutics, Brisbane, QLD 4076, Australia. ²Division of Cancer Research, Peter MacCallum Cancer Centre, Melbourne, VIC 3000, Australia. ${ }^{3}$ Sir Peter MacCallum Department of Oncology, University of Melbourne, Parkville, VIC 3052, Australia. ${ }^{4}$ Department of Surgery, Duke University Medical Center, Durham, North Carolina 27710, USA. ${ }^{5}$ Progen Pharmaceuticals, Brisbane, QLD 4076, Australia. ${ }^{6}$ Departments of Medicine and Immunology, Duke University Medical Center, Durham, North Carolina 27710, USA. ${ }^{7}$ Present address: Novasep, Kalkstrasse 218, 51377 Leverkusen, Germany. ${ }^{8}$ Present address: School of Humanities and Social Science, The University of Newcastle, Newcastle, NSW, Australia. 


\section{Received: 23 April 2018 Accepted: 21 May 2018}

Published online: 14 June 2018

\section{References}

1. Dredge K, Hammond E, Davis K, Li CP, Liu L, Johnstone K, et al. The PG500 series: novel heparan sulfate mimetics as potent angiogenesis and heparanase inhibitors for cancer therapy. Investig New Drugs. 2010;28:27683. https://doi.org/10.1007/s10637-009-9245-5.

2. Ferro V, Liu L, Johnstone KD, Wimmer N, Karoli T, Handley P, et al. Discovery of PG545: a highly potent and simultaneous inhibitor of angiogenesis, tumor growth, and metastasis. J Med Chem. 2012;55:3804-13. https://doi. org/10.1021/jm201708h.

3. Hammond E, Handley P, Dredge K, Bytheway I. Mechanisms of heparanase inhibition by the heparan sulfate mimetic PG545 and three structural analogues. FEBS Open Bio. 2013;3:346-51. https://doi.org/10. 1016/j.fob.2013.07.007.

4. Vlodavsky I, Singh P, Boyango I, Gutter-Kapon L, Elkin M, Sanderson RD, et al. Heparanase: from basic research to therapeutic applications in cancer and inflammation. Drug Resist Updat. 2016;29:54-75. https://doi.org/10. 1016/j.drup.2016.10.001.

5. Zhang Y-F, Tang X-D, Gao J-H, Fang D-C, Yang S-M. Heparanase: a universal immunotherapeutic target in human cancers. Drug Discov Today. 2011;16: 412-7. https://doi.org/10.1016/j.drudis.2011.02.015.

6. Ramani VC, Purushothaman A, Stewart MD, Thompson CA, Vlodavsky I, JL-S A, et al. The heparanase/syndecan-1 axis in cancer: mechanisms and therapies. FEBS J. 2013;280:2294-306. https://doi.org/10.1111/febs.12168.

7. Sanderson RD, Elkin M, Rapraeger AC, llan N, Vlodavsky I. Heparanase regulation of cancer, autophagy and inflammation: new mechanisms and targets for therapy. FEBS J. 2017;284:42-55. https://doi.org/10.1111/febs.13932.

8. Vlodavsky I, Gross-Cohen M, Weissmann M, llan N, Sanderson RD. Opposing functions of Heparanase-1 and Heparanase-2 in cancer progression. Trends Biochem Sci. 2018;43:18-31. https://doi.org/10.1016/j.tibs.2017.10.007.

9. Ostapoff KT, Awasthi N, Cenik BK, Hinz S, Dredge K, Schwarz RE, et al. $P G 545$, an angiogenesis and heparanase inhibitor, reduces primary tumor growth and metastasis in experimental pancreatic cancer. Mol Cancer Ther. 2013;12:1190-201. https://doi.org/10.1158/1535-7163.MCT-12-1123.

10. Boyango I, Barash U, Naroditsky I, Li J-P, Hammond E, llan N, et al. Heparanase cooperates with Ras to drive breast and skin tumorigenesis. Cancer Res. 2014 74:4504-14. https://doi.org/10.1158/0008-5472.CAN-13-2962.

11. Brennan TV, Lin L, Brandstadter JD, Rendell VR, Dredge $K$, Huang $X$, et al. Heparan sulfate mimetic PG545-mediated antilymphoma effects require TLR9-dependent NK cell activation. J Clin Invest. 2016;126:207-19. https:// doi.org/10.1172/JCI76566.

12. Hermano E, Meirovitz A, Meir K, Nussbaum G, Appelbaum L, Peretz T, et al. Macrophage polarization in pancreatic carcinoma: role of heparanase enzyme. J Natl Cancer Inst. 2014;106 https://doi.org/10.1093/jnci/dju332.

13. Khamaysi I, Singh $P$, Nasser $S, A$ wad $H$, Chowers $Y$, Sabo E, et al. The role of heparanase in the pathogenesis of acute pancreatitis: a potential therapeutic target. Sci Rep. 2017;7:715. https://doi.org/10.1038/s41598-017-00715-6.

14. Koliopanos A, Friess H, Kleeff J, Shi X, Liao Q, Pecker I, et al. Heparanase expression in primary and metastatic pancreatic cancer. Cancer Res. 2001;61: 4655-9. http://www.ncbi.nlm.nih.gov/pubmed/11406531

15. Hoffmann A-C, Mori R, Vallbohmer D, Brabender J, Drebber U, Baldus SE, et al. High expression of heparanase is significantly associated with dedifferentiation and lymph node metastasis in patients with pancreatic ductal adenocarcinomas and correlated to PDGFA and via HIF1a to HB-EGF and bFGF. J Gastrointest Surg. 2008;12:1674-81. https://doi.org/10.1007/ s11605-008-0628-2. 2

16. Hunter KE, Palermo C, Kester JC, Simpson K, Li J-P, Tang LH, et al. Heparanase promotes lymphangiogenesis and tumor invasion in pancreatic neuroendocrine tumors. Oncogene. 2014;33:1799-808. https://doi.org/10. 1038/onc.2013.142

17. Knudsen ES, Vail P, Balaji U, Ngo H, Botros IW, Makarov V, et al. Stratification of pancreatic ductal adenocarcinoma: combinatorial genetic, stromal, and immunologic markers. Clin Cancer Res. 2017;23:4429-40. https://doi.org/10. 1158/1078-0432.CCR-17-0162

18. Mattiola I, Pesant M, Tentorio PF, Molgora M, Marcenaro E, Lugli E, et al. Priming of human resting NK cells by autologous M1 macrophages via the engagement of IL-1 $\beta$, IFN- $\beta$, and IL-15 pathways. J Immunol. 2015;195:281828. https://doi.org/10.4049/jimmunol.1500325.
19. Mills $C D$, Lenz $L L$, Harris RA. A breakthrough: macrophage-directed cancer immunotherapy. Cancer Res. 2016;76:513-6. https://doi.org/10.1158/00085472.CAN-15-1737.

20. Kadowaki T, Shimada M, Inagawa H, Kohchi C, Hirashima M, Soma G-I. Reconsideration of macrophage and dendritic cell classification. Anticancer Res. 2012;32:2257-61. http://www.ncbi.n/m.nih.gov/pubmed/22641660

21. Dredge K, Hammond E, Handley P, Gonda TJ, Smith MT, Vincent C, et al. PG545, a dual heparanase and angiogenesis inhibitor, induces potent antitumour and anti-metastatic efficacy in preclinical models. Br J Cancer. 2011; 104:635-42. https://doi.org/10.1038/bjc.2011.11.

22. Hammond E, Brandt R, Dredge K. PG545, a heparan sulfate mimetic, reduces heparanase expression in vivo, blocks spontaneous metastases and enhances overall survival in the 4T1 breast carcinoma model. PLoS One. 2012;7:e52175. https://doi.org/10.1371/journal.pone.0052175.

23. Mondal S, Roy D, Camacho-Pereira J, Khurana A, Chini E, Yang L, et al. HSulf1 deficiency dictates a metabolic reprograming of glycolysis and TCA cycle in ovarian cancer. Oncotarget. 2015;6:33705-19. https://doi.org/10.18632/ oncotarget.5605

24. Shteingauz A, Boyango I, Naroditsky I, Hammond E, Gruber M, Doweck I, et al. Heparanase enhances tumor growth and Chemoresistance by promoting autophagy. Cancer Res. 2015;75:3946-57. https://doi.org/10.1158/ 0008-5472.CAN-15-0037.

25. Kundu S, Xiong A, Spyrou A, Wicher G, Marinescu VD, P-HD E, et al. Heparanase promotes glioma progression and is inversely correlated with patient survival. Mol Cancer Res. 2016;14:1243-53. https://doi.org/10.1158/ 1541-7786.MCR-16-0223.

26. Weissmann M, Arvatz G, Horowitz N, Feld S, Naroditsky I, Zhang Y, et al. Heparanase-neutralizing antibodies attenuate lymphoma tumor growth and metastasis. Proc Natl Acad Sci U S A. 2016;113:704-9. https://doi.org/10. 1073/pnas.1519453113.

27. Spyrou A, Kundu S, Haseeb L, Yu D, Olofsson T, Dredge K, et al. Inhibition of heparanase in pediatric brain tumor cells attenuates their proliferation, invasive capacity, and in vivo tumor growth. Mol Cancer Ther. 2017;16: 1705-16. https://doi.org/10.1158/1535-7163.MCT-16-0900.

28. Singh $P$, Blatt A, Feld S, Zohar $Y$, Saadi E, Barki-Harrington $L$, et al. The heparanase inhibitor PG545 attenuates colon cancer initiation and growth, associating with increased p21 expression. Neoplasia. 2017;19:175-84. https://doi.org/10.1016/j.neo.2016.12.001.

29. Winterhoff B, Freyer L, Hammond E, Giri S, Mondal S, Roy D, et al. PG545 enhances anti-cancer activity of chemotherapy in ovarian models and increases surrogate biomarkers such as VEGF in preclinical and clinical plasma samples. Eur J Cancer. 2015;51:879-92. https://doi.org/10.1016/j.ejca. 2015.02.007.

30. Jung D-B, Yun M, Kim E-O, Kim J, Kim B, Jung JH, et al. The heparan sulfate mimetic PG545 interferes with Wnt/ $\beta$-catenin signaling and significantly suppresses pancreatic tumorigenesis alone and in combination with gemcitabine. Oncotarget. 2015;6:4992-5004. https://doi.org/10.18632/ oncotarget.3214.

31. Dredge K, Brennan TV, Hammond E, Lickliter JD, Lin L, Bampton D, et al. A phase I study of the novel immunomodulatory agent PG545 (pixatimod) in subjects with advanced solid tumours. Br J Cancer. 2018; in press

32. Chen DS, Mellman I. Elements of cancer immunity and the cancer-immune set point. Nature. 2017:541:321-30. https://doi.org/10.1038/nature21349.

33. O'Donnell JS, Long GV, Scolyer RA, Teng MWL, Smyth MJ. Resistance to PD1/PDL1 checkpoint inhibition. Cancer Treat Rev. 2017;52:71-81. https:// doi.org/10.1016/j.ctrv.2016.11.007

34. Kim K, Skora AD, Li Z, Liu Q, Tam AJ, Blosser RL, et al. Eradication of metastatic mouse cancers resistant to immune checkpoint blockade by suppression of myeloid-derived cells. Proc Natl Acad Sci U S A. 2014;111: 11774-9. https://doi.org/10.1073/pnas.1410626111.

35. Narni-Mancinelli E, Chaix J, Fenis A, Kerdiles YM, Yessaad N, Reynders A, et al. Fate mapping analysis of lymphoid cells expressing the NKp46 cell surface receptor. Proc Natl Acad Sci U S A. 2011;108:18324-9. https://doi. org/10.1073/pnas.1112064108.

36. Coupland LA, Chong BH, Parish CR. Beware of NK cells in pre-clinical metastasis models. Clin Exp Metastasis. 2013;30:945-7. https://doi.org/10. 1007/s10585-013-9582-9.

37. Ribas A, Dummer R, Puzanov I, VanderWalde A, Andtbacka RHI, Michielin O, et al. Oncolytic Virotherapy promotes Intratumoral T cell infiltration and improves anti-PD-1 immunotherapy. Cell. 2017;170:1109-1119.e10. https:// doi.org/10.1016/j.cell.2017.08.027. 
38. van der Woude LL, Gorris MAJ, Halilovic A, Figdor CG, de Vries IJM. Migrating into the tumor: a roadmap for T cells. Trends Cancer. 2017;3:797808. https://doi.org/10.1016/j.trecan.2017.09.006.

39. Prendergast GC, Mondal A, Dey S, Laury-Kleintop LD, Muller AJ. Inflammatory reprogramming with IDO1 inhibitors: turning immunologically unresponsive "cold" tumors "hot". Trends Cancer. 2018;4:38-58. https://doi. org/10.1016/j.trecan.2017.11.005.

40. Betting DJ, Yamada RE, Kafi K, Said J, van Rooijen N, Timmerman JM. Intratumoral but not systemic delivery of CpG oligodeoxynucleotide augments the efficacy of anti-CD20 monoclonal antibody therapy against B cell lymphoma. J Immunother. 32:622-31. https://doi.org/10.1097/CJI. 0b013e3181ab23f1.

41. Jang JK, Khawli LA, Canter DC, Hu P, Zhu TH, Wu BW, et al. Systemic delivery of chTNT-3/CpG immunoconjugates for immunotherapy in murine solid tumor models. Cancer Immunol Immunother. 2016;65:511-23. https:// doi.org/10.1007/s00262-016-1813-x.

42. Spranger S, Gajewski T. Rational combinations of immunotherapeutics that target discrete pathways. J Immunother Cancer. 2013;1:16. https://doi.org/ 10.1186/2051-1426-1-16.

43. Ott PA, Hodi FS, Kaufman HL, Wigginton JM, Wolchok JD. Combination immunotherapy: a road map. J Immunother Cancer. 2017;5:16. https://doi. org/10.1186/s40425-017-0218-5.

44. Krieg AM. Toll-like receptor 9 (TLR9) agonists in the treatment of cancer. Oncogene. 2008;27:161-7. https://doi.org/10.1038/s.onc.1210911.

45. Wang S, Campos J, Gallotta M, Gong M, Crain C, Naik E, et al. Intratumoral injection of a CpG oligonucleotide reverts resistance to PD-1 blockade by expanding multifunctional CD8+ T cells. Proc Natl Acad Sci U S A. 2016;113: E7240-9. https://doi.org/10.1073/pnas.1608555113.

46. Sato-Kaneko F, Yao S, Ahmadi A, Zhang SS, Hosoya T, Kaneda MM, et al. Combination immunotherapy with TLR agonists and checkpoint inhibitors suppresses head and neck cancer. JCl Insight. 2017;2 https://doi.org/10. 1172/jci.insight.93397.

47. Sommariva M, Le Noci V, Storti C, Bianchi F, Tagliabue E, Balsari A, et al.

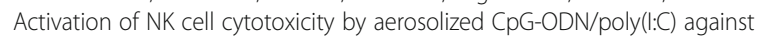
lung melanoma metastases is mediated by alveolar macrophages. Cell Immunol. 2017;313:52-8. https://doi.org/10.1016/j.cellimm.2017.01.004.

48. Dammeijer F, Lievense LA, Kaijen-Lambers ME, van Nimwegen M, Bezemer K, Hegmans JP, et al. Depletion of tumor-associated macrophages with a CSF-1R kinase inhibitor enhances antitumor immunity and survival induced by DC immunotherapy. Cancer Immunol Res. 2017;5:535-46. https://doi.org/ 10.1158/2326-6066.CIR-16-0309

49. Eissler N, Mao Y, Brodin D, Reuterswärd P, Andersson Svahn H, Johnsen Jl, et al. Regulation of myeloid cells by activated T cells determines the efficacy of PD-1 blockade. Oncoimmunology. 2016;5:e1232222. https://doi.org/10. 1080/2162402X.2016.1232222

50. Gad SC. Animal models in toxicology. 3rd ed. Boca Raton: CRC Press; 2015.

51. Heikenwalder M, Polymenidou M, Junt T, Sigurdson C, Wagner H, Akira S, et al. Lymphoid follicle destruction and immunosuppression after repeated CpG oligodeoxynucleotide administration. Nat Med. 2004;10:187-92. https:// doi.org/10.1038/nm987.

\section{Ready to submit your research? Choose BMC and benefit from:}

- fast, convenient online submission

- thorough peer review by experienced researchers in your field

- rapid publication on acceptance

- support for research data, including large and complex data types

- gold Open Access which fosters wider collaboration and increased citations - maximum visibility for your research: over $100 \mathrm{M}$ website views per year

At BMC, research is always in progress.

Learn more biomedcentral.com/submissions 